\title{
Correlação da produtividade do feijão com a resistência à penetração do solo sob plantio direto
}

\author{
Guilherme J. Carvalho ${ }^{1}$, Morel de P. Carvalho ${ }^{1}$, Onã da S. Freddi ${ }^{2} \&$ Mariana V. Martins ${ }^{1}$
}

\begin{abstract}
RESUMO
A resistência mecânica do solo à penetração (RP) exerce grande influência sobre o desenvolvimento vegetal, uma vez que o crescimento das raízes, tal como a produtividade das culturas, variam de forma inversamente proporcional ao seu valor. Portanto, o objetivo do trabalho foi avaliar a correlação entre a produtividade de feijão (PG) e a RP do Latossolo Vermelho cultivado a quatro anos no sistema plantio direto, pertencente a FEIS/UNESP. Os atributos RP e PG, foram determinados em uma malha regular contendo 119 pontos amostrais. A RP foi determinada nas camadas de 0-0,05, 0,05-0,10, 0,10-0,15, 0,15-0,20, 0,20-0,25 e 0,25-0,30 m. Os dados foram submetidos a análise estatística descritiva, assim como a correlação linear e geoestatística. A correlação linear entre a PG e a RP foi praticamente nula, uma vez que, em função das camadas estudadas do solo, apontou coeficientes de determinação $\left(R^{2}\right)$ menores que 0,03 e não significativos. A análise geoestatística apontou moderada estrutura de dependência espacial para RP nas camadas de 0,05-0,10 e 0,10-0,15 m e forte para a PG; entretanto, sua análise espacial conjunta mostrou-se sem correlação e, assim, a variabilidade espacial da RP não influenciou a da PG.
\end{abstract}

Palavras-chave: índice de cone, variabilidade espacial, geoestatística, compactação do solo

\section{Correlation between bean yield and soil penetration resistance under no-tillage}

\begin{abstract}
The soil mechanical resistance to penetration (PR) has great influence on vegetative development as the root growth and the crop productivity change in inverse proportion. Thus, the objective of this research was to study correlation between the bean grain productivity and the PR in an Oxisol cultivated for four years in no-tillage system at FEIS/UNESP. The attributes PR and yield were determined in a regular grid with 119 sample points. The PR was determined in the layers of $0-0.05,0.05-0.10,0.10-0.15,0.15-0.20$ and $0.25-0.30 \mathrm{~m}$. The results were submitted to procedures of descriptive statistics, linear correlation and geostatistic analysis. The linear correlation between the yield and PR was practically null, as in all soil layers investigated it showed determination coefficients $\left(\mathrm{R}^{2}\right)$ smaller than 0.03 and not significant. The geostatistic analysis showed moderate structure of spatial dependency for PR in the layers of 0.05-0.10 and 0.10-0.15 m, and strong for yield; however, the conjugate spatial analysis of such attributes showed no correlation, therefore, the spatial variability of PR did not influence the yield.
\end{abstract}

Key words: cone index, spatial variability, geostatistic, soil compaction

${ }^{1}$ FEIS/UNESP. Av. Brasil, Centro, 56, CP 31, CEP 15385-000, Ilha Solteira, SP. Fone: (18) 3743-1000. E-mail: carvalho gj@yahoo.com.br; morel@agr.feis.unesp.br ${ }_{2}^{2}$ FCAV/UNESP. Departamento de Solos e Adubos, Via de Acesso Prof. Paulo Donato Castellane s/n, CEP 14884-900, Jaboticabal, SP. Fone: (16) $3209-2672$. E-mail: ona_freddi@yahoo.com.br 


\section{INTRODUÇÃO}

A cultura do feijoeiro constitui-se numa das mais importantes explorações agrícolas do Brasil, não só pela área cultivada e pelo valor da produção como, também, por se tratar da principal fonte de proteína das camadas sociais de menor poder aquisitivo (Soratto, 2002). Juntamente com o arroz, milho e a mandioca, representam a base da alimentação humana; trata-se de uma importante cultura de inverno, utilizada tanto em sucessão quanto em rotação com outras, por proporcionar boa rentabilidade ao agricultor, além de implementar a geração de empregos (Muçouçah, 1994).

Na safra 2003/04, a produção nacional de feijão alcançou $3,2910^{9} \mathrm{~kg}$, ocupando uma área de aproximadamente 4,12 milhões de ha, sendo o rendimento médio de grãos muito baixo, ao redor de $780 \mathrm{~kg} \mathrm{ha}^{-1}$ (FAO, 2005), considerado baixo por ter a cultura um potencial para rendimento superior a $3500 \mathrm{~kg} \mathrm{ha}^{-1}$ (FNP Consultoria \& Comércio, 2003); e, contudo, em regiões nas quais a agricultura é mais tecnificada, como é o exemplo do Estado de São Paulo, o rendimento médio das três safras já ultrapassou $1200 \mathrm{~kg} \mathrm{ha}^{-1}$ (Camargo et al., 2001).

Nos últimos anos, métodos de preparo do solo, como o reduzido e o plantio direto, vêm sendo adotados em substituição aos convencionais. Segundo Tavares Filho et al. (2001), o sistema plantio direto provoca certa compactação superficial do solo, por estar associado ao tráfego de máquinas agrícolas, assim como ao não revolvimento do sistema. A compactação do solo é caracterizada por uma alteração estrutural que causa aumento da sua densidade e redução da porosidade total (Stone et al., 2002), que pode reduzir a penetração das raízes (Bengough et al., 1997), e alterar o equilíbrio na proporção de gases do solo e a disponibilidade de água e nutrientes às raízes das plantas (Stirzaker et al., 1996); em conseqüência, o funcionamento bioquímico da planta é alterado restringindo, entre outros fatores, a taxa fotossintética, o crescimento da parte aérea (Turner, 1997) e a produção da cultura (Beutler \& Centurion, 2004). Segundo Bowen \& Kratky (1985), os parâmetros mais usados para se avaliar a compactação são a densidade do solo e a resistência mecânica a penetração, sendo que está última possui boa correlação com o crescimento radicular, uma vez que a elongação das raízes varia de forma inversamente proporcional a tal resistência.

Valores de resistência mecânica à penetração variando entre 1,0 e 3,5 MPa (Merotto \& Mundstock, 1999), ou de 2,0 a 4,0 MPa, segundo Arshad et al. (1996), podem restringir, ou mesmo impedir, o crescimento e o desenvolvimento das raízes; já para o conceito de intervalo hídrico, Silva et al. (1994) relacionaram o valor de 2,0 MPa como limite crítico para o ótimo desenvolvimento radicular.

Ante a importância econômica da cultura do feijão, normalmente associada às baixas produtividades nacionais, os estudos científicos dos sistemas de produção e o avanço tecnológico para aumentar sua produtividade, competitividade e rentabilidade, são imprescindíveis. Ainda com a expansão generalizada do sistema plantio direto, o estudo sobre a que nele ocorre, assim como a determinação de valores a partir dos quais a sua produção é prejudicada, tor- nam-se fundamentais. Com o exposto, o objetivo do trabalho foi avaliar a correlação linear e a espacial entre a produtividade de grãos de feijão e a resistência mecânica à penetração de um Latossolo Vermelho cultivado no sistema plantio direto, durante quatro anos.

\section{MATERIAL E MÉTODOS}

O trabalho foi desenvolvido na Fazenda de Ensino e Pesquisa da Faculdade de Engenharia - FEIS/UNESP, Campus de Ilha Solteira, localizada no município de Selvíria, MS, na latitude $22^{\circ} 23^{\prime} \mathrm{S}$ e longitude $51^{\circ} 27^{\prime} \mathrm{W}$, com precipitação média anual de $1300 \mathrm{~mm}$ e temperatura média de $23,5^{\circ} \mathrm{C}$. O tipo climático é Aw, segundo a classificação de Köeppen, caracterizado como tropical úmido com estação chuvosa no verão e seca no inverno. O solo, classificado, segundo o Sistema Brasileiro de Classificação de Solos (EMBRAPA, 1999), foi um Latossolo Vermelho distroférrico típico argiloso, A moderado, hipodistrófico, álico, caulinítico, férrico, muito profundo e moderadamente ácido.

A área experimental, instalada sob pivô central, foi cultivada quatro anos no sistema plantio direto, com milho e/ou soja no verão e milho e/ou feijão no outono-inverno. Em 17/ 05/2003 semeou-se a cultivar Pérola, no espaçamento de 0,50 $\mathrm{m}$ na entrelinha e com 15 sementes por metro com uma semeadora de plantio direto. Na adubação de semeadura foram utilizados $200 \mathrm{~kg} \mathrm{ha}^{-1}$ da fórmula 08-28-16 e na de cobertura, realizada após 20 dias a emergência das plântulas, $200 \mathrm{~kg} \mathrm{ha}^{-1}$ da fórmula 20-00-20.

Para avaliação da variabilidade espacial montou-se uma malha retangular no espaçamento de 15 x 15 m estabelecida entre dois terraços utilizando-se, para isto, um nível ótico comum, totalizando 44 pontos; realizou-se, também, a adição de 75 pontos, espaçados 7,5 x 7,5 m visando detalhar o estudo da dependência espacial dos dados para condições menores que $15 \mathrm{~m}$, totalizando 119 pontos. Os atributos do solo pesquisados foram: resistência mecânica do solo à penetração (RP) e umidade gravimétrica (UG). O parâmetro da planta avaliado foi à produtividade de grãos (PG).

Em junho de 2003, trabalhando conforme metodologia da EMBRAPA (1979), foram extraídos os valores da RP nas camadas de 0-0,05 (RP1); 0,05-0,10 (RP2); 0,10-0,15 (RP3); 0,15-0,20 (RP4); 0,20-0,25 (RP5); 0,25-0,30 m (RP6) e a RP média de todas as camadas (RPM) com penetrômetro de impacto (Stolf, 1991). Concomitantemente, coletaram-se amostras de solo com um trado de rosca, para determinação da umidade gravimétrica do solo (UG), nas mesmas camadas que para a RP, para todos os pontos da malha; assim, ficaram representadas por UG1, UG2, UG3, UG4, UG5 e UG6 da superfície para a subsuperfície do solo e a UG média de todas as camadas (UGM). A expressão utilizada para o cálculo da RP, foi a seguinte:

$$
\mathrm{RP}=\{5,6+6,89[\mathrm{~N} /(\mathrm{P}-\mathrm{A}) 10]\} 0,0981
$$

donde: RP representou a resistência mecânica do solo a penetração, em MPa; $\mathrm{N}$ foi o número de impactos efetuados com 
o martelo para a obtenção da leitura, e A e P foram as leituras antes e após a realização dos impactos $(\mathrm{cm})$. Por outro lado, a PG foi obtida pela coleta das plantas contidas no entorno de cada ponto da malha. A área útil para coleta foi $4,00 \mathrm{~m}^{2}$ (2,0 x 2,0 m). A PG foi representada pela massa de grãos contida na área de coleta, corrigida para $13 \%$ de umidade e transformada para área equivalente a um hectare.

Os resultados foram submetidos aos procedimentos da estatística descritiva, para a obtenção da média, mediana, moda, valor máximo e mínimo, desvio padrão, coeficientes de variação, assimetria e curtose. Também se efetuou a análise de distribuição de freqüência dos dados, visando-se testar a hipótese de normalidade pelo teste de Shapiro \& Wilk (1965) a $1 \%$ de probabilidade. Discriminou-se o tipo indeterminado de distribuição de freqüências quando o atributo não resultou em uma distribuição normal, após lhe aplicar o teste de lognormalidade.

A análise de dependência espacial foi avaliada por meio das técnicas da geoestatística, em que o semivariograma assume papel fundamental na estimativa da dependência espacial entre amostras. Os valores do coeficiente de determinação $\left(\mathrm{R}^{2}\right)$ e da soma dos quadrados dos resíduos (SQR) foram utilizados na seleção inicial dos semivariogramas, assim como os do $\mathrm{R}^{2}$ da validação cruzada o foram ao final, conforme Vieira et al. (1983). O semivariograma foi estimado pela equação:

$$
\hat{\gamma}(h)=\frac{1}{2 N(h)} \sum_{i=1}^{N(h)}\left[Z\left(x_{i}\right)-Z\left(x_{i}+h\right)\right]^{2}
$$

donde: $\mathrm{N}(\mathrm{h})$ é o número de pares de valores medidos $\mathrm{Z}\left(\mathrm{x}_{\mathrm{i}}\right)$, $\mathrm{Z}\left(\mathrm{x}_{\mathrm{i}}+\mathrm{h}\right)$, separados pelo vetor $\mathrm{h}$.

Normalmente, o semivariograma é representado pelo gráfico de $\hat{\gamma}(h)$ versus $h$, ou seja, a semivariância do atributo versus distância (Vieira et al., 1997). Os semivariogramas forneceram estimativas dos parâmetros: efeito pepita $\left(\mathrm{C}_{0}\right)$, patamar $\left(\mathrm{C}_{0}+\mathrm{C}\right)$ e alcance. $\mathrm{O}$ efeito pepita $\left(\mathrm{C}_{0}\right)$ é o parâmetro do semivariograma que indica a variabilidade não explicada dos modelos, considerando a distância de amostragem utilizada (Cambardella et al., 1994). O semivariograma apresenta efeito pepita puro quando a semivariância $\hat{\gamma}(\mathrm{h})$ for igual para todos os valores de h. O patamar $\left(C_{0}+C\right)$ é o valor da semivariância onde a curva se estabiliza sobre um valor constante, sendo representado pelo ponto em que toda a semivariância da amostra é de influência aleatória (Trangmar et al., 1985). À medida que h aumenta, $\hat{\gamma}(\mathrm{h})$ também aumenta até um valor máximo no qual se estabiliza. Este valor no qual $\hat{\gamma}(\mathrm{h})$ se estabiliza, chama-se patamar, e é aproximadamente igual à variância dos dados (Vieira et al., 1997). O alcance (a) da dependência espacial representa a distância na qual os pontos amostrais estão correlacionados entre si. Portanto, os pontos localizados numa área de raio igual ao alcance possuem maior semelhança entre si, que aqueles localizados fora desta (Vieira et al., 1997). A análise da dependência espacial (ADE) foi efetuada segundo a expressão contida em Robertson (1998):

$$
\mathrm{ADE}=[\mathrm{C} /(\mathrm{C}+\mathrm{Co})] 100
$$

donde: ADE é o estimador da dependência espacial (\%); C a variância estrutural e $\mathrm{C}+\mathrm{C}_{0}$, o patamar. Testaram-se os modelos esférico, exponencial e gaussiano, cujo primeiro é dado por $\hat{\gamma}(\mathrm{h})=\left[1,5(\mathrm{~h} / \mathrm{a})-0,5(\mathrm{~h} / \mathrm{a})^{3}\right]$, para $0<\mathrm{h}<$ a e $\hat{\gamma}(\mathrm{h})=\mathrm{C}_{0}+\mathrm{C}$, para $\mathrm{h}>$ a. Em seguida à modelagem dos semivariogramas, realizou-se a interpolação por krigagem ordinária, sendo esta uma técnica de interpolação para estimativa de valores de uma propriedade em locais não amostrados, a partir de dezesseis vizinhos amostrados na malha. A krigagem, no entanto, faz uso de um interpolador linear não tendencioso e de variância mínima, que assegura a melhor estimativa dos dados não amostrados. Este estimador tem, como base, os dados amostrais da variável regionalizada e as propriedades estruturais do semivariograma obtido a partir de tais dados (Vieira et al., 1983). Na falta de estacionaridade para algum atributo, retirou-se a tendência dos dados pela técnica do refinamento pela mediana, conforme metodologia descrita em Silva et al. (2003), a seguir: a) os dados foram dispostos de acordo com sua posição original na malha amostral; b) foi calculada a mediana correspondente a cada linha e coluna, e c) foi subtraído, de cada valor amostrado, o valor da mediana da linha e da coluna em que se encontrou este valor, adicionando-lhe o valor da mediana de todo o conjunto de dados. Desta forma, pôdese calcular novamente o semivariograma com os resíduos obtidos deste procedimento; também se montou a matriz de correlação, objetivando-se efetuar as regressões lineares simples para as combinações, duas a duas, entre todos os atributos estudados (solo e planta). O objetivo foi estudar a correlação linear entre eles, na tentativa de procurar selecionar aqueles que provavelmente proporcionariam semivariograma cruzado, que é uma ferramenta geoestatística que permite verificar o relacionamento entre as variabilidades de duas séries de valores espacialmente medidos, tornando possível avaliar se a variabilidade de uma série é acompanhada pela da outra, de forma direta como indireta. Para duas funções aleatórias $Z_{1}$ e $Z_{2}$, o semivariograma cruzado é igual a:

$$
\gamma_{12}(h)=1 / 2 E\left[Z_{1}\left(x_{i}\right)-Z_{1}\left(x_{i}+h\right)\right]\left[Z_{2}\left(x_{i}\right)-Z_{2}\left(x_{i}+h\right)\right]
$$

entre valores amostrados a uma distância de separação h, sendo estimado por:

$$
\gamma_{12}^{*}(h)=\frac{1}{2 N(h)} \sum_{i-1}^{n(h)}\left[Z_{1}\left(x_{i}\right)-Z_{1}\left(x_{i}+h\right)\right]\left[Z_{2}\left(x_{I}\right)-Z_{2}\left(x_{i}+h\right)\right]
$$

sendo: N(h) o número de valores separados pelo vetor h; ao contrário do semivariograma, que é sempre positivo, o semivariograma cruzado pode assumir valores positivos, indicando que o aumento de uma das variáveis é acompanhado pelo aumento da outra. Quando negativos, indica que o aumento em uma das variáveis corresponde a um decréscimo da outra (Souza, 1992).

\section{RESULTADOS E DISCUSSÃO}

Para avaliar a variabilidade dos dados, analisaram-se os coeficientes de variação, conforme o critério de classificação 
proposto por Gomes (2000): baixo (CV < 10\%); médio $(10 \%<\mathrm{CV}>20 \%)$; alto $(20 \%<\mathrm{CV}>30 \%)$ e muito alto (CV $>30 \%$ ), o qual tem sido adotado em trabalhos relacionados a estudos de solo, por ser uma medida adimensional e possibilitar a comparação da variabilidade de duas variáveis. A variabilidade da RP na camada de $0-0,05 \mathrm{~m}$ foi muito alta (RP1); na camada de 0,05-0,15 m foi alta (RP2 e RP3), e entre 0,15-0,30 m foi média (RP4, RP5 e RP6) (Tabela 1). Observa-se diminuição da variabilidade da RP com o aumento da profundidade do solo. Provavelmente, a maior variabilidade encontrada nas camadas superficiais seja proporcionada pela ação das hastes sulcadoras para deposição do adubo e encontradas nas semeadoras de plantio direto.

Também se verifica (Tabela 1 ) que a profundidade $0,15 \mathrm{~m}$, em que a variabilidade passa de alta para média, é coincidente com a profundidade de trabalho da haste. Este efeito se torna mais acentuado uma vez que a área é irrigada com o pivô central, permitindo-se colher duas safras por ano; já para a umidade, a variabilidade foi média em todo o perfil estudado (de UG1 a UG6), com coeficientes de variação entre 19,8\% (UG1) e 15,4\% (UG3), indicando uma condição homogênea para coleta dos dados da RP, o que poderia influenciar os valores de RP encontrados. Por outro lado, a produtividade do feijão (PG) apresentou média variabilidade, com coeficiente de variação de 15,2\%.

O conhecimento da distribuição de freqüência dos dados de uma variável tem importantes conseqüências práticas, uma vez que os métodos de análise a serem aplicados são distintos para as diferentes distribuições. Exemplificando, sabe-se que a média é uma boa medida descritiva para dados com distribuição normal, se porém o mesmo não acontece àqueles com distribuição lognormal, aos quais a mediana se torna uma boa medida descritiva (Spiegel, 1985); assim, a aná- lise da Tabela 1 revelou que a UG4, RP3 e a PG, apresentaram distribuição normal, enquanto para a RP4, RP5 e RP6, foi lognormal, tendo os demais atributos (UG1, UG2, UG3, UG5, UG6, RP1 e RP6) o tipo indeterminado de distribuição de freqüência. Webster (1985) relatou que a normalidade dos dados não é uma exigência da geoestatística, sendo conveniente apenas que a distribuição não apresente caudas muito alongadas, o que poderia comprometer as análises. Por outro lado, Gonçalves et al. (2001) evidenciaram que a distribuição normal dos dados é muito interessante na avaliação da dependência espacial; no entanto, mais importante que a normalidade é a verificação de que tais dados não apresentem tendência.

Na Tabela 1, os valores médios da RP foram de 1,3; 2,3; 2,5; 2,8; 2,9 e 2,7 MPa, respectivamente, na camada de 0$0,30 \mathrm{~m}$. O valor da RP1 foi substancialmente menor que os restantes sendo, inclusive, menor que o valor de 2,0 $\mathrm{MPa}$ relacionado por Silva et al. (1994) devido, provavelmente, à maior quantidade de matéria orgânica presente na camada superficial do solo, resultante da decomposição da palhada da cultura anterior e também da ação dos mecanismos rompedores do solo utilizados para deposição do adubo e semente que a semeadora/adubadora de plantio direto possui. Em contrapartida, nas profundidades restantes (RP2 a RP6) os valores da referida resistência foram superiores àqueles de 2,0 MPa, denotando o clássico problema de compactação do solo que tal sistema proporciona. A maior compactação do solo foi observada na camada de 0,15-0,25 m. Em relação à umidade do solo, seus valores médios em profundidade, além de apresentarem pequena variação, ficaram próximos e ligeiramente menores que os representativos do valor da capacidade de campo, conforme Martins (2002) e que foi de $0,20 \mathrm{~kg} \mathrm{~kg}^{-1}$, sendo considerado ideal para a determinação de

Tabela 1. Análise descritiva da produtividade do feijão (PG), da resistência mecânica a penetração (RP) e da umidade gravimétrica (UG) do Latossolo Vermelho

\begin{tabular}{|c|c|c|c|c|c|c|c|c|c|c|}
\hline \multirow{3}{*}{ Atributo(a) } & \multicolumn{9}{|c|}{ Medidas Estatísticas Descritivas } & \multirow{3}{*}{$\begin{array}{c}\text { Distribuição } \\
\text { de } \\
\text { Freqüência(b) }\end{array}$} \\
\hline & \multirow{2}{*}{ Média } & \multirow{2}{*}{ Mediana } & \multirow{2}{*}{ Moda } & \multicolumn{2}{|c|}{ Valor } & \multirow{2}{*}{$\begin{array}{l}\text { Desvio } \\
\text { Padrão }\end{array}$} & \multicolumn{3}{|c|}{ Coeficiente } & \\
\hline & & & & Mínimo & Máximo & & Variação (\%) & Curtose & Assimetria & \\
\hline \multicolumn{11}{|c|}{ Umidade Gravimétrica } \\
\hline UG1 $\left(\mathrm{kg} \mathrm{kg}^{-1}\right)$ & 0,160 & 0,162 & 0,166 & 0,029 & 0,224 & $3,174.10^{-2}$ & 19,8 & 2,248 & $-1,036$ & I \\
\hline UG2 $\left(\mathrm{kg} \mathrm{kg}^{-1}\right)$ & 0,161 & 0,164 & 0,164 & 0,060 & 0,220 & $2,764.10^{-2}$ & 17,2 & 1,585 & $-0,879$ & I \\
\hline UG3 $\left(\mathrm{kg} \mathrm{kg}^{-1}\right)$ & 0,165 & 0,167 & 0,178 & 0,069 & 0,213 & $2,542.10^{-2}$ & 15,4 & 1,344 & $-0,817$ & I \\
\hline UG4 $\left(\mathrm{kg} \mathrm{kg}^{-1}\right)$ & 0,165 & 0,168 & 0,167 & 0,093 & 0,238 & $2,723.10^{-2}$ & 16,5 & 0,002 & $-0,270$ & $\mathrm{~N}$ \\
\hline UG5 $\left(\mathrm{kg} \mathrm{kg}^{-1}\right)$ & 0,171 & 0,172 & 0,189 & 0,091 & 0,271 & $3,013.10^{-2}$ & 17,6 & 1,345 & 0,008 & I \\
\hline UG6 $\left(\mathrm{kg} \mathrm{kg}^{-1}\right)$ & 0,172 & 0,174 & 0,157 & 0,051 & 0,237 & $3,182.10^{-2}$ & 18,5 & 1,865 & $-0,954$ & I \\
\hline UGM $^{1}$ & 0,167 & 0,168 & 0,167 & 0,092 & 0,235 & $2,503.10^{-2}$ & 15,0 & 0,340 & $-0,331$ & $\mathrm{~N}$ \\
\hline \multicolumn{11}{|c|}{ Resistência Mecânica do Solo à Penetração } \\
\hline RP1 (MPa) & 1,286 & 1,225 & 1,901 & 0,549 & 2,577 & $5,066 \cdot 10^{-1}$ & 39,4 & $-0,714$ & 0,220 & 1 \\
\hline RP2 (MPa) & 2,277 & 2,336 & 2,239 & 0,549 & 4,334 & $6,670.10^{-1}$ & 29,3 & 0,765 & $-0,180$ & I \\
\hline RP3 (MPa) & 2,510 & 2,481 & 2,481 & 1,000 & 3,929 & $5,643.10^{-1}$ & 22,5 & 0,295 & 0,060 & $\mathrm{~N}$ \\
\hline RP4 (MPa) & 2,792 & 2,738 & 3,253 & 1,676 & 4,605 & $5,227.10^{-1}$ & 18,7 & 1,110 & 0,801 & L \\
\hline RP5 (MPa) & 2,869 & 2,802 & 2,802 & 1,676 & 4,334 & $5,101 \cdot 10^{-1}$ & 17,8 & 0,097 & 0,589 & $\mathrm{~L}$ \\
\hline RP6 (MPa) & 2,677 & 2,674 & 2,802 & 1,676 & 3,929 & $4,347.10^{-1}$ & 16,2 & $-0,049$ & 0,422 & $\mathrm{~L}$ \\
\hline RPM & 2,408 & 2,384 & 2,352 & 1,507 & 3,478 & $3,583.10^{-1}$ & 14,9 & 0,603 & 0,269 & N \\
\hline \multicolumn{11}{|c|}{ Produtividade de Grãos } \\
\hline$P G\left(t^{h} a^{-1}\right)$ & 1,679 & 1,650 & 1,277 & 1,102 & 2,153 & $2,552 \cdot 10^{-1}$ & 15,2 & $-0,738$ & 0,018 & $\mathrm{~N}$ \\
\hline
\end{tabular}

(a) UG e RP, de 1 a 6, são, respectivamente, a umidade gravimétrica e a resistência a penetração em profundidade no solo; UGM e RPM são os valores médios dos referidos atributos, e PG é a produtividade de grãos do feijão, e (b) sendo N, L e I respectivamente as distribuições de frequêencia do tipo normal, lognormal e indeterminada 
tal resistência neste solo, não interferindo na análise da RP que é inversamente influenciada pela umidade do solo (Gerard et al., 1972), permitindo a comparação de tal resistência entre as camadas do solo. Por outro lado, conforme Fahl et al. (1998), o dado médio da produtividade de grãos de feijão $\left(1,679 \mathrm{t} \mathrm{ha}^{-1}\right)$ situou-se dentro dos limites do rendimento nacional do feijão irrigado, mas ficou bem abaixo do potencial de produtividade da cultura de $3500 \mathrm{~kg} \mathrm{ha}^{-1}$.

Na Tabela 2 as correlações estabelecidas entre as umidades gravimétricas apresentaram os valores extremos do coeficiente de correlação, de 0,863 a 0,497, respectivamente para as regressões entre a UGM versus UG4 e UG2 versus UG5. Da mesma forma, as correlações estabelecidas entre as resistências mecânicas apresentaram os valores extremos do coeficiente de correlação de 0,760 a 0,005, respectivamente para as regressões entre a RPM versus logRP3 e logRP6 versus RP2; já para as correlações estabelecidas entre as umidades gravimétricas e as resistências mecânicas, os referidos valores se mantiveram entre 0,171 e 0,001, respectivamente para as regressões entre a UG1 versus RP1 e UGM versus RPM.

Por outro lado, as correlações entre as umidades gravimétricas e a produtividade de grãos, oscilaram entre 0,132 e -0,002, respectivamente para UG1 versus PG e UG4 versus PG, ao passo que aquelas entre as resistências mecânicas e a referida produtividade permaneceram com os valores ínfimos de -0,089 e -0,013, respectivamente entre logRP5 versus PG e logRP6 versus PG (Tabela 2).

Supunha-se que os elevados valores de RP, encontrados na área e relacionados na Tabela 1, apresentassem correlação inversa com a PG, corroborando com a baixa produtividade encontrada para o feijão irrigado; no entanto, este ajuste, especificamente efetuado entre a PG e as RPs, apresentou um coeficiente de correlação não superior ao não significativo valor de $-0,089$, conforme pode ser visto na matriz de correlação, estabelecida para a regressão logRP5 versus PG (Tabela 2).
Na Tabela 3, a análise geoestatística evidenciou que apenas os atributos RP2, RP3 e PG apresentaram dependência espacial, comprovada pelos parâmetros dos semivariogramas. Em relação às sete UGs, todas apresentaram tendência, extraídas com a técnica do refinamento pela mediana. Desta forma, as UG1, UG2, UG3, UG4, UG5, UG6, UGM, RP1, logRP4, logRP5, logRP6 e RPM apresentaram ausência de dependência espacial, denotada pelo efeito pepita puro. Os valores do efeito pepita variaram entre 4,780 x $10^{-4}$ a $9,260 \times 10^{-4}\left[(\mathrm{~kg} \mathrm{~kg})^{-2}\right]$ para a umidade gravimétrica, e entre 3,256 x $10^{-3}$ a 2,570 x $10^{-1}\left[(\mathrm{MPa})^{2}\right]$ para a resistência mecânica a penetração. Assim, tanto a RP quanto a PG mostraram que a distribuição desses atributos no espaço não é aleatória, uma vez que suas classes de dependência espacial variaram de 61,3\% (moderada para a RP2 e RP3) a $80,0 \%$ (forte para a PG). Esses valores foram relativamente superiores aos obtidos por Gonçalves et al. (1998) e Souza et al. (2001), os quais foram, respectivamente, de 26 a 58\% e 50 a 53\%, mas inferiores aos de Carvalho et al. (2002), que foram de 73 a $90 \%$, Portanto, pôde-se constatar, no presente estudo, que $80 \%$ da variação total da PG foram explicados pela dependência espacial. Para todos os atributos que apresentaram dependência espacial, o modelo ajustado foi o esférico. Com relação ao alcance da dependência espacial, a resistência mecânica apresentou valores entre 64,5 (RP3) e 67,1 m (RP2), ao passo que a produtividade de grãos (PG) o apresentou com um valor de 20,2 $\mathrm{m}$. Tais valores foram substancialmente superiores aos de Gonçalves et al. (1998), que variaram entre 10 e $18 \mathrm{~m}$ e também superiores em relação aos de Souza et al. (2001) e Carvalho et al. (2002), os quais trabalharam com o mesmo solo do presente estudo, obtendo alcances variando respectivamente entre 13,1 e 58,8 m e entre 14,4 e 15,2 m. Essas diferenças provavelmente são originadas devido ao espaçamento dos pontos nas malhas amostrais. Segundo Trangmar et al. (1985), o alcance depende do tamanho da área amostrada e da escala de observação, sendo tanto maior quanto maior o intervalo entre medidas.

Tabela 2. Matriz de correlação entre a produtividade do feijão (PG), a resistência mecânica a penetração (RP) e a umidade gravimétrica (UG) do Latossolo Vermelho

\begin{tabular}{|c|c|c|c|c|c|c|c|c|c|c|c|c|c|c|}
\hline \multirow{2}{*}{ Atributo } & \multicolumn{14}{|c|}{ Coeficiente de Correlação } \\
\hline & UG1 & UG2 & UG3 & UG4 & UG5 & UG6 & UGM1 ${ }^{1}$ & RP1 & RP2 & RP3 & $\log \mathrm{RP} 4$ & $\log R P 5$ & $\log R P 6$ & $\mathrm{RPM}^{2}$ \\
\hline UG2 & $0,682^{*}$ & & & & & & & & & & & & & \\
\hline UG3 & $0,707^{\star}$ & $0,735^{\star}$ & & & & & & & & & & & & \\
\hline UG4 & $0,708^{*}$ & $0,708^{*}$ & $0,794^{*}$ & & & & & & & & & & & \\
\hline UG5 & $0,510^{*}$ & $0,497^{*}$ & $0,589^{*}$ & $0,629 *$ & & & & & & & & & & \\
\hline UG6 & $0,633^{*}$ & $0,698^{*}$ & $0,693^{*}$ & $0,703^{*}$ & $0,515^{\star}$ & & & & & & & & & \\
\hline UGM $^{1}$ & $0,798^{*}$ & $0,775^{*}$ & $0,815^{*}$ & $0,863^{*}$ & $0,758^{*}$ & $0,769^{*}$ & & & & & & & & \\
\hline RP1 & $0,171^{*}$ & $0,039^{\text {ns }}$ & $0,096^{\text {ns }}$ & $0,106^{\mathrm{ns}}$ & $0,038^{\mathrm{ns}}$ & $0,147^{\text {ns }}$ & $0,062^{\text {ns }}$ & & & & & & & \\
\hline RP2 & $0,161^{*}$ & $-0,030^{\mathrm{ns}}$ & $0,089^{\text {ns }}$ & $0,084^{\text {ns }}$ & $0,073^{\text {ns }}$ & $0,112^{\text {ns }}$ & $0,093^{\text {ns }}$ & $0,544^{*}$ & & & & & & \\
\hline RP3 & $0,004^{\mathrm{ns}}$ & $-0,153^{\text {ns }}$ & $-0,094^{\mathrm{ns}}$ & $-0,043^{\text {ns }}$ & $-0,088^{\text {ns }}$ & $-0,029^{\text {ns }}$ & $-0,106^{\mathrm{ns}}$ & $0,405^{*}$ & $0,634^{*}$ & & & & & \\
\hline $\log \mathrm{RP} 4$ & $-0,039^{\text {ns }}$ & $-0,057^{\mathrm{ns}}$ & $0,025^{\text {ns }}$ & $-0,016^{\text {ns }}$ & $-0,116^{\mathrm{ns}}$ & $-0,008^{\text {ns }}$ & $-0,033^{\text {ns }}$ & $0,189 *$ & $0,221^{*}$ & $0,517^{\star}$ & & & & \\
\hline $\log$ RP5 & $-0,025^{\mathrm{ns}}$ & $-0,048^{\mathrm{ns}}$ & $0,052^{\text {ns }}$ & $0,014^{\mathrm{ns}}$ & $-0,027^{\mathrm{ns}}$ & $-0,056^{\mathrm{ns}}$ & $0,003^{\text {ns }}$ & $-0,012^{\mathrm{ns}}$ & $0,070^{\text {ns }}$ & $0,244^{*}$ & $0,517^{*}$ & & & \\
\hline $\log R P 6$ & $0,047^{\mathrm{ns}}$ & $-0,002^{\mathrm{ns}}$ & $0,102^{\mathrm{ns}}$ & $0,064^{\text {ns }}$ & $0,051^{\text {ns }}$ & $-0,066^{\mathrm{ns}}$ & $0,052^{\text {ns }}$ & $0,062^{\text {ns }}$ & $0,005^{\text {ns }}$ & $0,128^{\mathrm{ns}}$ & $0,267^{*}$ & $0,652^{*}$ & & \\
\hline $\mathrm{RPM}^{2}$ & $0,068^{\mathrm{ns}}$ & $-0,083^{\text {ns }}$ & $0,064^{\mathrm{ns}}$ & $0,052^{\text {ns }}$ & $-0,053^{\text {ns }}$ & $0,012^{\mathrm{ns}}$ & $0,001^{\text {ns }}$ & $0,589 *$ & $0,621^{*}$ & $0,760^{\text {ns }}$ & $0,654^{*}$ & $0,577^{*}$ & $0,490^{*}$ & \\
\hline $\mathrm{PG}$ & $0,132^{\mathrm{ns}}$ & $0,070^{\mathrm{ns}}$ & $0,034^{\mathrm{ns}}$ & $-0,002^{\text {ns }}$ & $-0,068^{\mathrm{ns}}$ & $0,014^{\mathrm{ns}}$ & $0,065^{\mathrm{ns}}$ & $0,025^{\text {ns }}$ & $-0,035^{n s}$ & $-0,052^{\text {ns }}$ & $-0,082^{\text {ns }}$ & $-0,089^{\text {ns }}$ & $-0,013^{\text {ns }}$ & $-0,030^{\text {ns }}$ \\
\hline
\end{tabular}

(a) UG e RP, de 1 a 6 são, respectivamente, a umidade gravimétrica e a resistência mecânica a penetração em profundidade do solo; UGM e RPM são os valores médios dos referidos atributos, e PG é a produtividade de grãos do feijão, e $e^{(b) *}$ significativo a $5 \%$ de probabilidade, ns não significativo 
Tabela 3. Parâmetros dos semivariogramas ajustados à produtividade do feijão (PG) e a resistência mecânica a penetração do Latossolo Vermelho, nas camadas de 0,10-0,20 m (RP2) e 0,20-0,30 m (RP3)

\begin{tabular}{|c|c|c|c|c|c|c|c|c|}
\hline \multirow[t]{2}{*}{ Atributo } & \multicolumn{8}{|c|}{ Parâmetros do Semivariograma } \\
\hline & Modelo & $\begin{array}{l}\text { Efeito Pepita } \\
\text { (Co) }\end{array}$ & $\begin{array}{l}\text { Patamar } \\
\left(\mathrm{Co}_{0}+\mathrm{C}\right)\end{array}$ & $\begin{array}{c}\text { Variância } \\
\text { Estrutural (C) }\end{array}$ & Alcance (m) & $\mathbf{R}^{2}$ & $\operatorname{SQR}^{(a)}$ & $A D E(b)$ \\
\hline RP2 (MPa) & Esférico & $6,820.10^{-2}$ & $1,864.10^{-1}$ & $1,182.10^{-1}$ & 67,100 & 0,881 & $1,891.10^{-3}$ & 63,4 \\
\hline $\mathrm{RP3}(\mathrm{MPa})$ & Esférico & $6,480.10^{-2}$ & $1,676.10^{-1}$ & $1,028.10^{-1}$ & 64,500 & 0,952 & $4,166.10^{-4}$ & 61,3 \\
\hline$P G\left(t h a^{-1}\right)$ & Esférico & $9,600.10^{-3}$ & $4,810.10^{-2}$ & $3,850.10^{-2}$ & 20,2 & 0,798 & $7,883 \cdot 10^{-5}$ & 80,0 \\
\hline
\end{tabular}

(a) SQR - soma dos quadrados dos residuos; (b) ADE - avaliador da dependência espacial

A validação cruzada é uma ferramenta destinada a avaliar modelos alternativos de semivariogramas que efetuaram a krigagem. Na sua análise, cada ponto contido dentro do domínio espacial é removido individualmente, sendo seu valor estimado através da krigagem, como se não existisse. Desta forma, pode-se construir um gráfico de valores estimados versus medidos para todos os pontos. Um ajuste perfeito teria um coeficiente de regressão igual a um e a linha do melhor ajuste coincidiria com o modelo perfeito, isto é, com o coeficiente linear (a) igual a zero e o angular (b) igual a um (Robertson, 1998). Portanto, os modelos dos semivariogramas ajustados para realização da krigagem apresentaram-se de forma excelente, uma vez que os coeficientes lineares (a) e angulares (b) dos ajustes efetuados variaram entre 0,028 (PG) e 0,288 (RP3) para o a, e 0,884 (RP2 e RP3) e 0,986 (PG) para o b; logo, todos os parâmetros estudados apresentaram funções positivas e crescentes, com valores estimados próximos aos observados. Constatou-se também que o ajuste do semivariograma da PG foi substancialmente superior ao das RPs, uma vez que o valor do seu coeficiente linear (a) foi o que mais se aproximou de zero.

Ainda que a PG e a RP2 e RP3, quando analisados individualmente, não tenham variado de forma aleatória, isto é, tenham apresentado, individualmente, excelente padrão de variabilidade espacial, quando analisados conjuntamente seus padrões espaciais não foram concordantes e, portanto, não inter-relacionados, porque, como exemplo, ora baixos valores de resistência coincidiram com altos valores de produtividade, ora altos valores de resistência coincidiram tanto com altos valores de produtividade, como com baixos valores, o que pôde ser confirmado pela não obtenção do semivariograma cruzado entre os atributos (Figura 1). Freddi et al. (2005) não verificaram diferença na produtividade de feijão quando cultivado num Latossolo Vermelho irrigado por pivô central, no sistema plantio direto e preparo convencional do solo; segundo Kluthcouski et al. (2000) as culturas da soja e do feijão apresentam melhor adaptabilidade ao sistema plantio direto que o milho e, principalmente, que o arroz. Nota-se, pelos mapas de krigagem, que apenas $14,6 \%$ da área da RP2 e 10,2\% da RP3 apresentaram RP abaixo de 2 MPa e somente 5,9\% da área apresentaram produtividade acima de $1900 \mathrm{~kg} \mathrm{ha}^{-1}$; logo, a baixa produtividade da cultura não foi explicada pela elevada RP, proporcionada pelo não revolvimento do solo no sistema plantio direto e, sim, por outros fatores não analisados por ocasião do presente estudo (Figura 1).

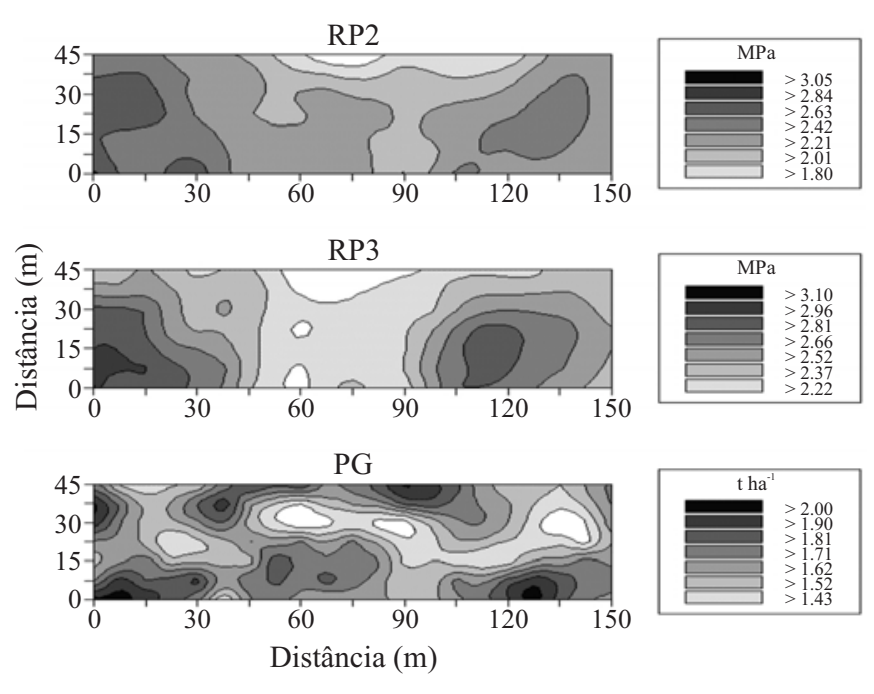

Figura 1. Mapas de krigagem da resistência mecânica do Latossolo Vermelho a penetração, nas camadas de 0,10-0,20 m (RP2) e 0,20-0,30 m (RP3), e da produtividade de grãos do feijão (PG)

\section{CONCLUSÕES}

1. A resistência mecânica à penetração, na camada 0,05 $0,15 \mathrm{~m}$, e da produtividade de grãos de feijão apresentou moderada e forte dependência espacial, respectivamente, com alcances de aproximadamente $60 \mathrm{~m}$ para a resistência mecânica do solo e $20 \mathrm{~m}$ para a produtividade.

2. Não houve correlação linear nem espacial entre a resistência mecânica do solo à penetração e a produtividade de grãos de feijão.

3. Valores de resistência mecânica do solo à penetração variando entre 1,29 a 2,87 MPa não restringiram a produtividade de grãos da cultura do feijão.

\section{LITERATURA CITADA}

Arshad, M. A.; Lowery, B.; Grossman, B. Physical tests for monitoring soil quality. In: Doran, J. W.; Jones, A. J. Methods for assessing soil quality. Madison: Soil Science Society of America, 1996. p.123-141. SSSA Special publication, 49

Bengough, A. G.; Croser, C.; Pritchard, J. A biophysical analysis of root growth under mechanical stress. Plant and Soil, Dordrecht, v.189, n.1, p.155-164, 1997.

Beutler, A. N.; Centurion, J. F.; Silva, A. P. Intervalo hídrico ótimo e a produção de soja e arroz em dois latossolos. Irriga, Botucatu, v.9, n.2, p.181-192, 2004. 
Bowen, J. E.; Kratky, B. A. Compactation del suelo. Agricultura de las Américas, v.34, n.1, p.10-14, 1985.

Cambardella, C. A.; Moorman, T. B.; Novak, J. M.; Parkin, T. B.; Karlen, D. L.; Turco, R. F.; Konopka, A. E. Field-scale variability of soil properties in Central Iowa Soils. Soil Science Society American Journal, Madison, v.58, n.5, p.1501-1511, 1994.

Camargo, A. M. M. P.; Caser, D. V.; Francisco, V. L. F. S. Previsões e estimativas das safras agrícolas no estado de São Paulo. Informações Economicas, v.31, n.2, p.101-117, 2001.

Carvalho, M. P.; Soratto, R. P.; Freddi, O. S. Variabilidade espacial de atributos físicos em um Latossolo Vermelho Distrófico sob preparo convencional em Selvíria (MS). Acta Scientiarum, Maringá, v.24, n.5, p.1353-1361, 2002.

EMBRAPA - Empresa Brasileira de Pesquisa Agropecuária. Serviço Nacional de Levantamento e Conservação de Solos. Manual de métodos de análise de solo. Rio de Janeiro: SNLCS, 1979. sp.

EMBRAPA - Empresa Brasileira de Pesquisa Agropecuária. Centro Nacional de Pesquisa de Solos. Sistema brasileiro de classificação de solos. Rio de Janeiro: SNLCS, 1999. 412p.

Fahl, J. I.; Camargo, M. B. P.; Pizzinatto, M. A.; Betti, J. A.; Melo, A. M. T.; De Maria, I. C.; Furlani, A. M. C. Instruções agrícolas para as principais culturas econômicas. 6 ed. Campinas: Instituto Agronômico, 1998. p.281-282. Boletim Técnico 200

FNP Consultoria \& Comércio. Feijão. In: Nakamae, I. J. (ed.). Agrianual 2003. São Paulo: Argos, 2003. cap. 30, p.345-354.

Freddi, O. S.; Carvalho, M. P.; Centurion, J. F.; Barbosa, G. F. Variabilidade espacial da produtividade de feijão em um Latossolo Vermelho distroférrico sob preparo convencional e plantio direto. Acta Scientiarum, Maringá, v.27, n.1, p.61-67, 2005.

FAO. Produção agrícola. http://www.fao.org/faostat/ collections?subset=agriculture. 28 Dez. 2005.

Gerard, C. J.; Mehth, H. C.; Hinojosa, F. Root growth in a clay soil. Soil Science, Baltimore, v.114, n.1, p.37-49, 1972.

Gomes, P. F. Curso de estatística experimental. 14.ed. Piracicaba: Degaspari, 2000. 477p.

Gonçalves, A. C. A.; Folegatti, M. V.; Mata, J. D. V. Análises exploratória e geoestatística da variabilidade de propriedades físicas de um Argissolo Vermelho. Acta Scientiarum, Maringá, v.23, n.5, p.1149-1157, 2001.

Gonçalves, A. C. A.; Mata, J. D. V.; Vieira, S. R.; Folegatti, M. V. Variabilidade espacial de produtividade e de resistência à penetração em área irrigada, sob dois sistemas de preparo. In: Avances en el Manejo del Suelo y agua en la Ingeniería Rural Latinoamericana, 18, 1998, La Plata. Resumos... La Plata: UNLP, 1998. p.113-119.

Kluthcouski, J.; Fancelli, A. L.; Dourado-Neto, D.; Ribeiro, M.; Ferraro, L. A. Manejo do solo e o rendimento de soja, milho, feijão e arroz em plantio direto. Scientia Agrícola, Piracicaba, v.57, n.1, p.97-104, 2000.

Martins, C. B. Análise da resistência mecânica à penetração de um Latossolo Vermelho Distrófico em função do grau de umidade. Ilha Solteira: UNESP, 2002, 46p. Trabalho Graduação

Merotto, A.; Mundstock, C. M. Wheat root growth as affected by soil strength. Revista Brasileira de Ciência Solo, Viçosa, v.23, n.2, p.197-202, 1999.

Muçouçah, M. J. Influência de cultivares de feijoeiro, adubação e inseticidas sobre a população e danos de Empoasca kraemeri (Ross \& Moore, 1957) (Hemiptera-Cicadelidae) e Bemisia tabaci (Gennadius, 1889) (Hemíptera-Aleyrodidae), em três épocas de semeadura. Jaboticabal: UNESP, 1994. 198p. Dissertação Mestrado
Robertson, G. P. GS ${ }^{+}$: Geostatistics for the environmental sciences. Michigan: Gamma Desing Software, 1998. 151p.

Shapiro, S. S.; Wilk, M. B. An analysis of variance test for normality: complete samples. Biometrika, London, v.52, n.5, p.591-611, 1965.

Silva, A. P.; Kay, B. D.; Perfect, E. Characterization of the least limiting water range. Soil Science Society of America Journal, Madison, v.58, n.6, p.1775-1784, 1994.

Silva, V. R.; Reichert, J. M.; Storck, L.; Feijó, S. Variabilidade espacial das características químicas do solo e produtividade de milho em um Argissolo Vermelho-Amarelo Distrófico arênico. Revista Brasileira de Ciência do Solo, Viçosa, v.27, n.6, p.1013-1020, 2003.

Soratto, R. P. Resposta do feijoeiro ao preparo do solo, manejo de água e parcelamento da adubação nitrogenada. Ilha Solteira: UNESP, 2002. 72p. Dissertação Mestrado

Souza, L. S. Variabilidade espacial do solo em sistemas de manejo. Porto Alegre: UFRGS, 1992. 162p. Tese Doutorado

Souza, Z. M.; Silva, M. L. S.; Guimarães, G. L.; Campos, D. T. S.; Carvalho, M. P.; Pereira, G. T. Variabilidade espacial de atributos físicos em um Latossolo Vermelho distrófico sob semeadura direta em Selvíria (MS). Revista Brasileira de Ciência do Solo, Viçosa, v.25, n.3, p.699-707, 2001.

Spiegel, M. R. Estatística. 2.ed. São Paulo: McGraw-Hill, 1985. 454p.

Stirzaker, R. J.; Passioura, J. B.; Wilms, Y. Soil structure and plant growth: Impact of bulk density and biopores. Plant and Soil, Dordrecht, v.185, n.1, p.151-162, 1996.

Stolf, R. Teoria e teste experimental de fórmulas de transformação dos dados de penetrômetro de impacto em resistência do solo. Revista Brasileira de Ciência do Solo, Viçosa, v.15, n.3, p.229-235, 1991.

Stone, L. F.; Guimarães, C. M.; Moreira, A. A. J. Compactação do solo na cultura do feijoeiro. I: efeitos nas propriedades físico-hídricas do solo. Revista Brasileira de Engenharia Agrícola e Ambiental, Campina Grande, v.6, n.2, p.207-212, 2002.

Trangmar, B. B.; Yost, R. S.; Uehara, G. Application of geostatistics to spatial studies of soil properties. Advances in Agronomy, San Diego, v.38, n.1, p.45-94, 1985.

Tavares Filho, J.; Barbosa, G. M. C.; Guimarães, M. F.; Fonseca, I. C. B. Resistência do solo à penetração e desenvolvimento do sistema radicular do milho (Zea mays L.) sob diferentes sistemas de manejo em um Latossolo Roxo. Revista Brasileira de Ciência do Solo, Viçosa, v.25, n.3, p.725-730, 2001.

Turner, N. C. Further progress in crop water relations. Advances in Agronomy, San Diego, v.58, n.1, p.293-325, 1997.

Vieira, S. R.; Hatfield, J. L.; Nielsen, D. R.; Biggar, J. W. Geostatistical theory and application to variability of some agronomical properties. Hilgardia, Berkeley, v.51, n.3, p.175, 1983.

Vieira, S. R.; Tillotson, P. M.; Biggar, J. W.; Nielsen, D. R. Scaling of semivariograms and the kriging estimation of field-measured properties. Revista Brasileira de Ciência do Solo, Viçosa, v.21, n.4, p.525-33, 1997.

Webster, R. Quantitative spatial analyses of soil in field. New York: Springer-Verlag, 1985. p.56. 